
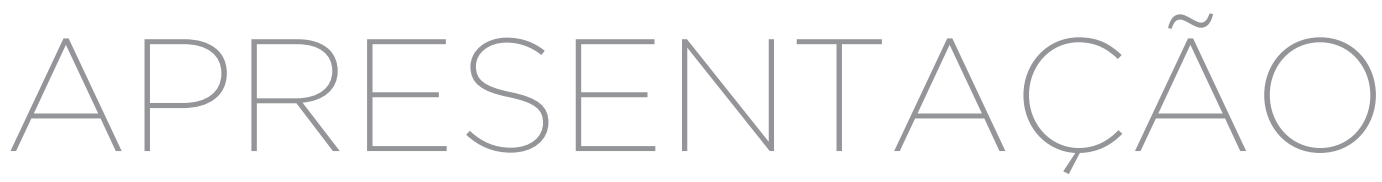

\title{
USOS E EFEITOS DAS AVALIAÇÕES EXTERNAS COMO OBJETO DE PESQUISA
}

Desde o início dos anos 1990, tem-se verificado o aumento e a consolidação de sistemas de avaliação de desempenho discente no Brasil, promovidos tanto pelo governo federal como por iniciativa de secretarias estaduais e municipais de educação. A consolidação das avaliações externas se desdobra em diversos mecanismos de política educacional, desde a estratégia da ampla divulgação de resultados aferidos ao conjunto da sociedade - notadamente através das coberturas jornalísticas em tom alarmante - até a premiação de agentes escolares em função dos resultados obtidos pelos alunos, dentre outros critérios. Em meio a diversas ações feitas em nome das avaliações externas e dado o peso que a divulgação de seus resultados acarreta, cabe investigar, de forma mais detida, o impacto dessas avaliações na orientação e no aperfeiçoamento do ensino e em que medida elas têm se tornado também instrumentos relevantes e pertinentes para a atuação de professores, gestores escolares e setores técnicos das secretarias de educação.

O presente número de Estudos em Avaliação Educacional reúne um conjunto de artigos que analisam diferentes indícios de impacto de avaliações externas na gestão educacional, tanto no cotidiano e na gestão de unidades escolares como no desempenho discente de redes ou sistemas educacionais. Destaca-se assim a relevância dessa agenda de pesquisa que focaliza a influência e a apropriação dos resultados desse tipo de avaliação em escolas e sistemas de ensino público brasileiros como um objeto de pesquisa que merece atenção de pesquisadores e profissionais da área com diferentes abordagens de investigação e possibilidades de aportar conhecimento sobre o tema. 
De fato, a adoção de avaliações externas que tenham como ponto forte o emprego de testes padronizados em larga escala, baseada em censo ou população de alunos (KELLAGHAN; GREANEY; MURRAY, 2011, p. 19) vai muito além da aplicação de provas, divulgação ou mesmo da análise dos resultados aferidos. O considerável volume de recursos públicos empregados e a crescente quantidade e qualidade das informações geradas impõem a necessidade política, particularmente no Brasil, de aprofundar e diversificar as pesquisas em torno das ações que utilizam as avaliações externas, como resposta responsável da comunidade acadêmica. Por outro lado, a frequente vinculação das avaliações externas à noção de accountability e a políticas de responsabilização pode provocar consequências indesejáveis à qualidade do trabalho escolar e ao caráter profissional de seus agentes institucionais (HAERTEL; HERMAN, 2005; LINN, 2000). Desse ponto de vista, reforça-se o interesse na investigação de eventuais efeitos dessas avaliações enquanto subsídio eminentemente pedagógico e formativo para agentes escolares e gestores educacionais. Ressalta-se, assim, que as eventuais implicações dos instrumentos de avaliação externa utilizados, seus desdobramentos e as expectativas nele depositadas demandam a verificação de sua validade consequencial (VIANNA, 2003) a partir dos efeitos que os resultados podem despertar no pensamento, nas atitudes e na ação dos diversos interessados. ${ }^{1}$

Atualmente, o crescente incentivo ao uso das avaliações por escolas e professores (GIMENES et al., 2013) parece transcender a compreensão das avaliações externas como instrumentos de gestão restritos ao âmbito dos diferentes órgãos decisórios do sistema educacional, como pode fazer supor a primeira geração de avaliação no Brasil, segundo classificação estabelecida por Bonamino e Sousa (2012). Para as autoras, a característica eminente das avaliações daquela primeira geração - como o Sistema de Avaliação da Educação Básica (Saeb) - ${ }^{2}$ seria a de propiciar o diagnóstico da qualidade da educação oferecida no Brasil objetivada em uma amostra representativa de escolas e redes de ensino com os desempenhos de seus respectivos alunos, o que inviabilizava consequências diretas para as unidades escolares ou seus profissionais.
1 Segundo Heraldo Vianna (2003, p. 54) a validade consequencial refere-se "ao impacto da avaliação sobre o sistema, determinando mudanças de pensamento, gerando novos comportamentos, formando novas atitudes e promovendo novas ações. A validade consequencial reflete em que medida a avaliação faz realmente alguma diferença para a comunidade".

2 Sem dúvida, o Saeb tem servido de parâmetro para a formulação de diversos sistemas de avaliação em larga escala, em especial quanto à apropriação de suas matrizes de avaliação e a utilização da abordagem metodológica referente à Teoria da Resposta ao Item - TRI. 
Além do Saeb, diversos sistemas de avaliação que compõem o atual cenário educacional apresentam como objetivo precípuo fomentar algum tipo de consequência direta para escolas e/ou para seus profissionais ao aferir e produzir resultados por unidade escolar. Tendo em vista esse desenho de avaliação externa, de caráter censitário, podemos encontrar dois grupos distintos. O primeiro deles seria composto pelas avaliações de segunda geração, vinculadas a consequências de caráter mais simbólico explicitadas pela ampla divulgação dos resultados à sociedade, pais de alunos e, evidentemente, pelos próprios agentes escolares (BONAMINO; SOUSA, 2012). Trata-se de um tipo de política de avaliação comumente chamada, na literatura acadêmica, de low stakes ou de responsabilização branda (BROOKE, 2006). A Prova Brasil, de iniciativa do Ministério da Educação, seria um exemplo de avaliação externa de segunda geração.

Já as avaliações de terceira geração seriam características das chamadas políticas de responsabilização forte ou high stakes e envolveriam não apenas a divulgação dos resultados aferidos, mas também sanções ou recompensas em função dos desempenhos obtidos por alunos e escolas. Uma ação de gestão nessa perspectiva de avaliação implicaria algum tipo de consequência material para as unidades escolares e docentes (BROOKE, 2008). No contexto específico da gestão pública brasileira, essa perspectiva de avaliação pode ser exemplificada nos processos de bonificação ou premiação de escolas e professores, verificados em diversos estados e municípios, e que apresentam como um de seus critérios os resultados obtidos pelo conjunto de alunos avaliados em uma mesma escola.

Em síntese, as avaliações de segunda e terceira geração estariam atreladas, de alguma forma, à noção de responsabilização de escolas e de seus agentes institucionais em relação aos resultados mensurados por avaliações externas. Sem descartar os aspectos políticos e ideológicos naturalmente vinculados à temática, é de extrema relevância investigar empiricamente algumas hipóteses, como: as políticas de incentivo material a professores e escolas, ou mesmo determinados tipos de exposição pública de resultados, 
podem estimular de alguma forma os profissionais de ensino a se engajarem na busca de melhores resultados no contexto brasileiro? Tais medidas seriam capazes de desestimular os agentes públicos da educação ao invés de motivá-los?

As políticas de responsabilização partem da hipótese de que os gestores dos sistemas e as redes de ensino, bem como as equipes gestoras de escolas e os professores, podem utilizar os dados gerados pelas avaliações externas e outros indicadores educacionais para tomar decisões estratégicas e modificar práticas administrativas e pedagógicas para alcançar melhores resultados educacionais, incrementando o desempenho dos estudantes. Obviamente a perspectiva não é culpar os agentes escolares pelos resultados inferiores ao adequado, mas estabelecer sua responsabilidade perante a sociedade pelo trabalho que desenvolvem nos estabelecimentos de ensino e suscitar a reflexão sobre suas práticas para analisar se "podem fazer algo diferente do que vêm fazendo" (FERNANDES; GREMAUD, 2009, p. 222). Na mesma direção, parece também importante discutir a responsabilidade dos gestores dos sistemas e das redes de ensino, considerando que integra suas competências prover as condições de funcionamento das escolas, seja no que diz respeito à infraestrutura, seja no que se refere às condições para o trabalho de docentes e gestores escolares, viabilizando as reflexões e mudanças almejadas para melhorar a qualidade dos serviços educacionais ofertados à população.

Também no plano internacional, a preocupação com a questão da efetiva utilidade das avaliações externas e políticas de responsabilização para melhorar os resultados educacionais tem gerado um crescente número de pesquisas (MARSH; McCOMBS; MARTORELL, 2010; STILLMAN, 2011; SLAVIN et al., 2012; MANDINACH; GRUMMER, 2013), muitas das quais têm focalizado as percepções dos agentes sobre as políticas e as apropriações das informações geradas e disponibilizadas. De fato, a compreensão e o uso dos dados das avaliações externas pelos agentes escolares possibilitam sua intervenção como protagonistas no processo, usando efetivamente os dados como insumo estratégico para a tomada de decisões. 
O estudo de Mandinach e Grummer (2013) nos EUA, por exemplo, ressalta que, apesar de a ênfase nas políticas que utilizam dados ser crescente naquele país, ainda se confere pouca atenção para a formação dos profissionais em torno do uso dos dados (data use). As autoras enfatizam a necessidade de desenvolver a capacidade de tomar decisões baseadas nos dados disponíveis (Data-Driven Decision Making $D D D M)$ e propõem processos de formação de profissionais de educação com esse objetivo, realçando a relevância de sua “alfabetização técnica” para o uso dos dados (data literacy). Marsh, McCombs e Martorell (2010) examinaram estratégias de "treinamento" com monitores para o DDDM em escolas na Flórida (instructional coaching), concluindo que a melhor compreensão dos professores sobre os dados contribui para a melhoria do ensino e o desempenho dos alunos. Stillman (2011), por sua vez, chega a conclusões semelhantes, mas enfatiza a influência dos fatores contextuais, especialmente o papel do diretor ou sua liderança, formação docente em serviço comprometida com a mudança e melhoria dos resultados escolares. Segundo a autora, quando os diretores proporcionam aos professores oportunidades de formação alinhadas com a política e valorizam o papel destes como protagonistas de mudanças na escola a partir das práticas de sala de aula, gera-se uma "tensão produtiva" que favorece seu desenvolvimento profissional e seu engajamento na perspectiva da mudança de práticas docentes com vistas à melhoria dos resultados escolares.

No cenário brasileiro, determinados usos de avaliações externas, protagonizados por Secretarias de Educação (BROOKE; CUNHA, 2011), podem gerar efeitos diversos dependendo das intenções e objetivos políticos da gestão central dos sistemas de ensino, das características e do histórico da rede escolar, do perfil socioeconômico do alunado e do próprio sentido que adquirem tais ações ao longo do tempo. Em especial, quando se constata serem as escolas - e seus agentes - instâncias capazes de reação e reinterpretação das políticas e ações externas a elas direcionadas (MARTINS, 2008) e não apenas unidades executoras da orientação de uma gestão central. 
De todo modo, as formas como as escolas e seus profissionais reagem à divulgação dos resultados e às diferentes políticas de responsabilização praticadas no país ainda é assunto pouco explorado empiricamente. Isto é, no âmbito das consequências das políticas de avaliação externa no Brasil, ainda pouco se conhece em relação aos seus efeitos, sobretudo quanto ao trabalho realizado por escolas e professores, mesmo sendo essa uma alegada preocupação da gestão educacional, em âmbito federal, estadual ou municipal.

O Departamento de Pesquisa Educacionais da Fundação Carlos Chagas, por meio de seu Núcleo de Estudos de Avaliação, tem realizado estudos e pesquisas sobre o eventual uso das avaliações externas, bem como analisado a literatura produzida no campo nas últimas duas décadas (SILVA et al., 2013; GIMENES et al., 2013; BAUER; REIS, 2014) no intuito de mapear e aprofundar essa temática. Visando a um maior intercâmbio e uma ampliação do debate em relação aos possíveis efeitos das avaliações externas no cotidiano escolar e na gestão educacional, o dossiê deste número apresenta alguns resultados de pesquisas realizados pelo grupo de pesquisa interdisciplinar e interinstitucional Observatório Educação e Cidade, ${ }^{3}$ que tem estudado as desigualdades de oportunidades educacionais, o mapeamento de indicadores educacionais e a apropriação dos sistemas de informação e avaliação no estado do Rio de Janeiro. Trata-se de uma articulação que reúne pesquisadores de diversas instituições (PUC-Rio, UFRJ e Uerj) e de diferentes áreas do conhecimento. Desde 2007 o grupo vem desenvolvendo vários projetos de pesquisa com fomento da Faperj, Inep, CNPq e Capes. O dossiê traz três textos com resultados de pesquisas desse grupo, desenvolvidas com diferentes abordagens metodológicas.

Em novembro de 2013, o Observatório Educação e Cidade realizou um seminário para discutir os resultados acumulados, sobretudo no que se refere às políticas de avaliação e responsabilização e as alterações que vêm provocando no cenário educacional e na gestão das redes de ensino e das escolas. Nessa oportunidade, foi possível estreitar laços de colaboração com pesquisadores da Universidade Federal de Juiz de Fora (UFJF) que desenvolvem investigações sobre
3 Os trabalhos apresentados foram realizados no período de 2011 a 2014 , com apoio ao Projeto "Desigualdades de Oportunidades Educacionais e Dimensões da Alfabetização da Educação Básica no Estado do Rio de Janeiro" coordenado pela Universidade Federal do Rio de Janeiro (UFRJ), por meio do Edital n. 038/2010/Capes/Inep - Programa Observatório da Educação. 
4 Os pesquisadores integram

Projeto "Análise da evolução da Educação Básica no Brasil a partir dos indicadores de fluxo e proficiência", apoiado pela Coordenação de Aperfeiçoamento de Pessoal de Níve Superior - Capes, por meio do Edita n. 038/2010/Capes/Inep - Programa Observatório da Educação. a evolução da educação básica a partir dos indicadores de fluxo e proficiência. ${ }^{4} \mathrm{O}$ primeiro artigo que integra o dossiê traz resultados das pesquisas realizadas por esse grupo no âmbito dos sistemas estaduais de ensino. Fernando Tavares Júnior e Luiz Flávio Neubert discutem, nesse artigo, a evolução recente das políticas de avaliação no Brasil, realçando o protagonismo dos estados nesse processo. Os autores chamam a atenção para a disseminação crescente de avaliações estaduais anuais, censitárias e relacionadas à gestão de suas redes na última década. $\mathrm{O}$ trabalho analisa a associação entre a implantação de Sistemas Estaduais de Avaliação Educacional no Brasil e o desenvolvimento da proficiência média entre os alunos das respectivas redes estaduais de ensino. Para isso, os autores utilizam resultados agregados das redes estaduais nas avaliações nacionais, como parâmetro externo. Os autores concluem que há uma associação estatisticamente significativa entre a implantação de avaliações nos estados e a elevação da proficiência média nos anos seguintes.

Três dos artigos apresentados trazem resultados de pesquisas realizadas na rede municipal do Rio de Janeiro. $\mathrm{O}$ artigo de Cynthia Paes de Carvalho, Ana Cristina Prado de Oliveira e Maria de Fátima Magalhães de Lima apresenta três estudos, articulando análises de cunho qualitativo e quantitativo sobre dados da rede municipal do Rio de Janeiro: observações e entrevistas realizadas em uma escola pública da rede e análises de dados dos questionários contextuais dos diretores da Prova Brasil 2007, 2009 e 2011. As autoras discutem os desafios e tensões no âmbito da gestão escolar no contexto das políticas de avaliação e responsabilização. Destacam em particular a sobrecarga de trabalho burocrático numa situação frequente de precariedade de condições de administração, a mediação tensa que as equipes de gestão precisam realizar junto aos agentes escolares para efetivar a implementação das políticas, e, ainda, as tendências de mudança na gestão pedagógica para promover melhores resultados escolares nas avaliações externas. O estudo propõe a necessidade de maior reflexão sobre a pesquisa e a formação em gestão escolar frente aos desafios políticos e pedagógicos no contexto atual. 
Com base em dados coletados em grupos focais realizados com gestores escolares, membros da gestão central e técnicos dos órgãos intermediários de gestão da rede municipal de educação do Rio de Janeiro, o artigo de Rodrigo Rosistolato, Ana Pires do Prado e Silvina Julia Fernández descreve e analisa as percepções de profissionais da gestão sobre as avaliações externas de aprendizagem e seus reflexos no cotidiano escolar. Os autores identificam uma tendência à utilização dos dados das avaliações como orientadores das práticas de gestão, mas também observam lacunas de conhecimento sobre os aspectos mais técnicos das avaliações. Essa dinâmica produz o que os autores classificaram como "efeito dominó": um conjunto de cobranças sucessivas que induzem, simultaneamente, estratégias de gestão que visam à melhoria do desempenho institucional e "jeitinhos" para ampliar os índices das escolas.

Mariane Campelo Koslinski, Carolina Portela da Cunha e Felipe Macedo de Andrade investigam o perfil das escolas que foram premiadas por um dos programas que compõem a política de accountability escolar da Secretaria Municipal de Educação do Rio de Janeiro (SME/RJ). Partindo da revisão bibliográfica nacional e internacional que indica a relevância dos desenhos de políticas de responsabilização e as atitudes ou cursos de ação que podem induzir nos agentes escolares, os autores descrevem a política de responsabilização escolar da SME/RJ e discutem alguns de seus efeitos com base em dados da própria Secretaria e dos questionários contextuais da Prova Brasil. Dessa forma, identificam algumas limitações do programa de responsabilização e tecem considerações sobre algumas alterações ocorridas na regulamentação no período que buscam corrigir alguns dos sinais/incentivos emitidos pelo programa estudado.

Espera-se, assim, contribuir para a discussão sobre as políticas de avaliação e responsabilização levadas a cabo em redes públicas brasileiras e propiciar a discussão e a reflexão sobre a pluralidade de percepções e reações que geram nos agentes educacionais no âmbito das unidades escolares. Acredita-se que tal reflexão, lastreada por dados de pesquisa empírica, possa também favorecer iniciativas de formação 
dos profissionais da educação para que se responsabilizem como protagonistas tecnicamente competentes e comprometidos com a democratização efetiva do direito de aprender das crianças e jovens brasileiros.

VANDRÉ GOMES DA SILVA vgomes@fcc.org.br

CYNTHIA PAES DE CARVALHO cynthiapaesdecarvalho@puc-rio.br

\section{REFERÊNCIAS}

BAUER, Adriana; REIS, Adriana Teixeira. Base de Dados Avaliação: balanço da produção teórica sobre avaliação de sistemas educacionais no Brasil: 1988 a 2011. São Paulo: Fundação Carlos Chagas, 2014. Disponível em: <http://acervo.fcc.org.br>. Acesso em: nov. 2014.

BONAMINO, Alicia; SOUSA, Sandra Zákia. Três gerações de avaliação da educação básica no Brasil: interfaces com o currículo da/na escola. Educação e Pesquisa, São Paulo, v. 38, n. 2, jun. 2012.

BROOKE, Nigel. O futuro das políticas de responsabilização educacional no Brasil. Cadernos de Pesquisa, São Paulo, v. 36, n. 128, p. 377-401, maio/ago. 2006. Disponível em: <http://www.scielo.br/pdf/cp/v36n128/v36n128a06. pdf>. Acesso em: nov. 2014.

. Responsabilização Educacional no Brasil. Revista Iberoamericana de Evaluación Educativa, Madrid, v. 1, n. 1, p. 93-109, 2008.

BROOKE, Nigel; CUNHA, Maria Amália de A. A avaliação externa como instrumento da gestão educacional nos estados. Estudos \& Pesquisas Educacionais, São Paulo, n. 2, p. 17-79, nov. 2011.

FERNANDES, Reynaldo; GREMAUD, Amaury Patrick. Qualidade da educação: avaliação, indicadores e metas. In: VELOSO, Fernando et al. (Org.). Educação Básica no Brasil: construindo um país do futuro. Rio de Janeiro: Elsevier, 2009. p. 213-238.

GIMENES, Nelson et al. Além da Prova Brasil: investimento em sistemas próprios de avaliação externa. Estudos em Avaliação Educacional, São Paulo, v. 24, n. 55, p. 12-32, ago. 2013. 
HAERTEL, Edward G.; HERMAN, Joan. A Historical Perspective on Validity Arguments for Accountability Testing. In: HAERTEL, E. G.; HERMAN, J. (Ed.). Uses and Misuses of data for educational Accountability and Improvement: The $104^{\text {th }}$ Yearbook of the National Society for Study of Education, Part. 2, J. L. Herman and E. H. Haertel (Org.). Malden MA: Blackwell, 2005. p. 1-34.

KELLAGHAN, Thomas; GREANEY, Vicent; MURRAY, T. Scott. 0 uso dos resultados da avaliação de desempenho educacional. Rio de Janeiro: Elsevier, 2011.

LINN, Robert L. Assessments and Accountability. Educational Researcher, v. 29, n. 2, p. 4-16, 2000.

MANDINACH, Ellen; GRUMMER, Edith. A Systemic View of Implementing Data Literacy in Educator Preparation. Educational Researcher, v. 42, p. 30-37, jan./fev. 2013. Disponível em: < http://edr.sagepub.com/content/42/1/30. short>. Acesso em: nov. 2014.

MARSH, Julie A.; McCOMBS, Jennifer Sloan; MARTORELL, Francisco. How Instructional Coaches Support Data-Driven Decision Making. Educational Policy, v. 24, n. 6, p. 872-907, nov. 2010. Disponível em: <http://epx.sagepub. com>. Acesso em: nov. 2014.

MARTINS, Angela. Aspectos organizacionais e dinâmicos na gestão da escola pública: dilemas e conflitos. Revista Brasileira de Política e Administração da Educação, v. 24, p. 135-152, 2008.

SILVA, Vandré Gomes et al. Uso da avaliação externa por equipes gestoras e profissionais docentes: um estudo em quatro redes de ensino público. São Paulo: FCC/SEP, 2013. Disponível em: <http://www.fcc.org.br/biblioteca/publicacoes/textos_fcc/arquivos/38/arquivoAnexado.pdf $>$. Acesso em: nov. 2014.

SLAVIN, Robert E. et al. Effects of a Data-Driven District Reform Model on State Assessment Outcomes. American Educational Research Journal, v. XX, n. 10, p. 1-26, 2012. Disponível em: <http://aer.sagepub.com/content/50/2/371.full. pdf + html>. Acesso em: nov. 2014.

STILLMAN, Jamy. Teacher Learning in an Era of High-Stakes Accountability: Productive Tension and Critical Professional Practice. Teachers College Record, v. 113, n. 1, p. 133-180, jan. 2011.

VIANNA, Heraldo Marelim. Avaliações nacionais em larga escala: análises e propostas. Estudos em Avaliação Educacional, São Paulo, n. 27, p. 41-76, jan.jjul. 2003. 